\title{
Invasive growth of Aspergillus oryzae in rice koji and increase of nuclear number
}

\section{CURRENT STATUS: UNDER REVISION}

Fungal Biology and Biotechnology $\triangle B M C$

Mizuki Yasui

University of Tsukuba

Ken Oda

National research institute of Brewing

Shunsuke Masuo

University of Tsukuba

Takuya Katayama

University of Tokyo

Jun-ichi Maruyama

University of Tokyo

Naoki Takaya

University of Tsukuba

Norio Takeshita

Tsukuba Daigaku

ఐ takeshita.norio.gf@u.tsukuba.ac.jpCorresponding Author

ORCiD: https://orcid.org/0000-0003-2666-4991

DOI:

$10.21203 / \mathrm{rs} .2 .22172 / \mathrm{v} 1$

\section{SUBJECT AREAS}

Biotechnology and Bioengineering

\section{KEYWORDS}

Aspergillus oryzae, koji, rice, nuclei, mitosis 
Abstract

'Rice koji' is a solid culture of Aspergillus oryzae on steamed rice grains. Multiple parallel fermentation, wherein saccharification of rice by $A$. oryzae and alcohol fermentation by the budding yeast occur simultaneously, lead to the formation of a variety of ingredients of Japanese sake. In sake brewing, the degree of mycelial invasive growth into the steamed rice, called 'haze-komi', highly correlates with the digestibility and quality of rice $k o j i$, since the hyphae growing into the rice secrete amylases and digest starch of rice. In this study, we investigated mycelial distribution of GFPtagged $A$. oryzae in rice koji made with different types of rice, such as sake rice and eating rice, with 50 or $90 \%$ polishing rate to remove abundant proteins and lipids near the surface. In addition, we compared transcriptomes of $A$. oryzae in the different types of rice koji. Finally, we found that $A$. oryzae increases the nuclear number and hyphal width in the course of 1-3 days cultivation. That characteristic could be corelated with the secretory capacity of several enzymes, and be an important selectively acquired characteristic during the breeding in long history.

Full Text

Due to technical limitations, full-text HTML conversion of this manuscript could not be completed. However, the manuscript can be downloaded and accessed as a PDF.

Figures 
A C; Chiyo-nishiki (eating rice) Y; Yamada-nishiki (sake rice)
90; polished $10 \%$ surface of rice

50; polished $50 \%$ (for top-quality sake, daiginjo-shu)

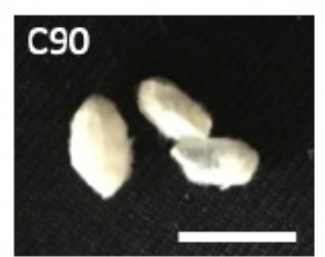

B
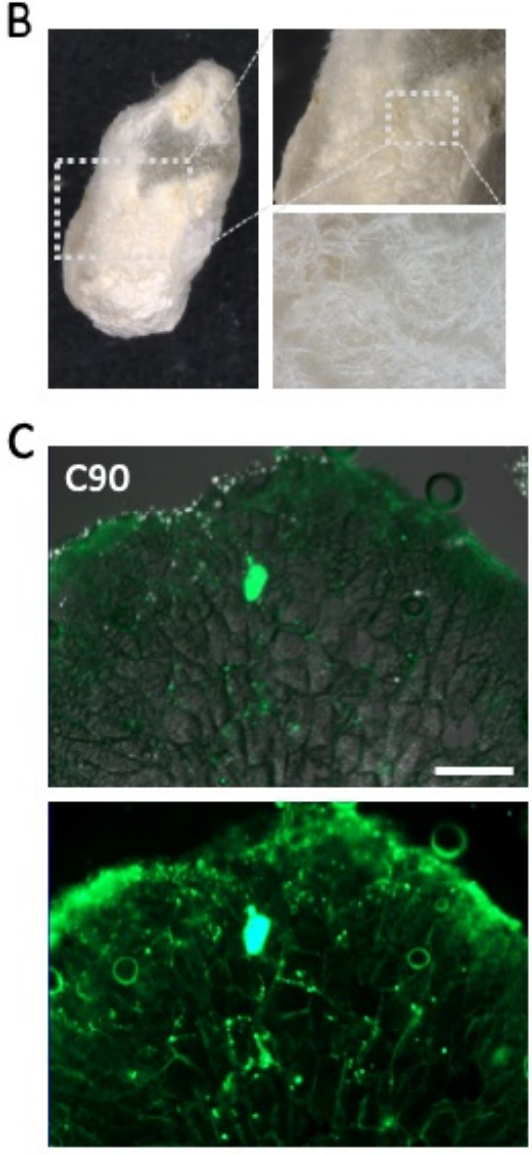
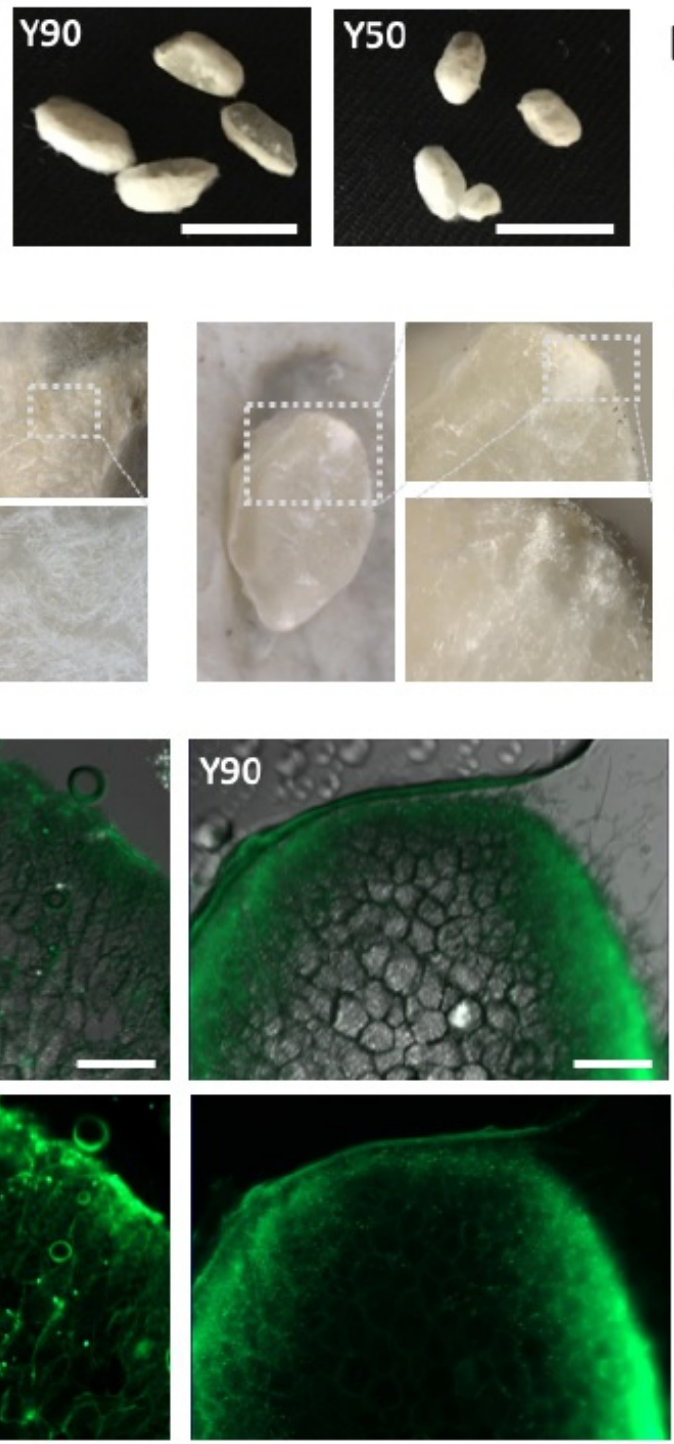
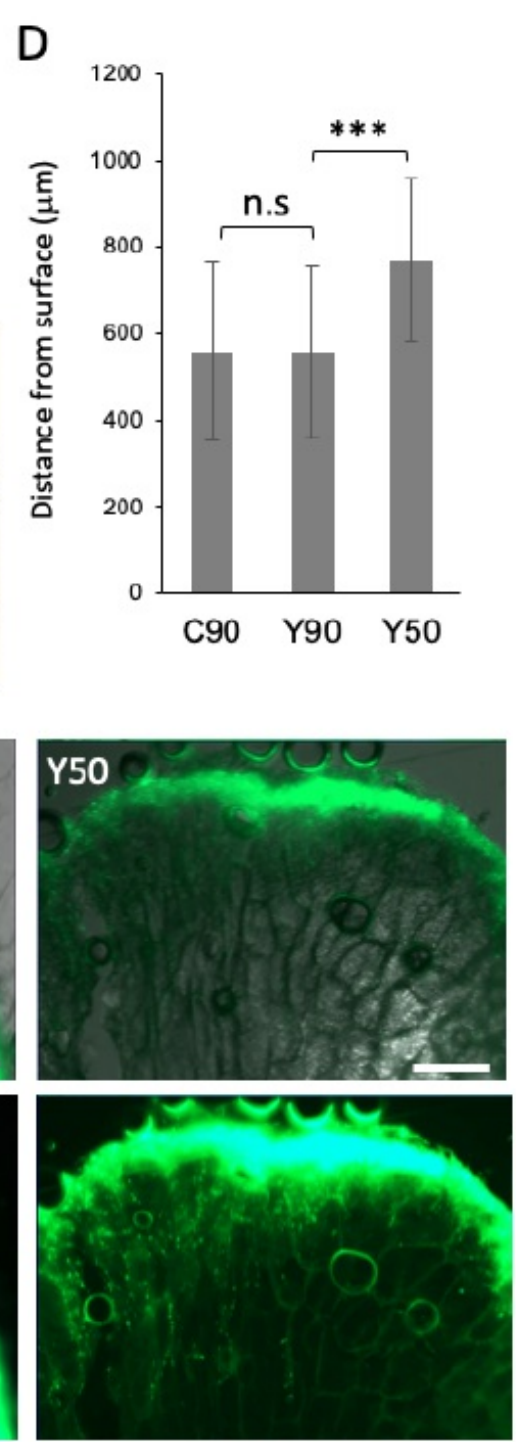

Figure 1

Imaging analysis of $A$. oryzae penetration into steamed rice. (A) Koji of different rice races and polishing rates. Chiyo-nishiki (eating rice) and Yamada-nishiki (sake rice) polished 90\% or $50 \%$. Scale bar: $1 \mathrm{~cm}$. (B) Images of section of koji by zoom microscopy. (C) Fluorescent images of A. oryzae (H2B-GFP) penetration into the steamed rice. The sections were sliced by cryo-microtome. Scale bars: $200 \mu \mathrm{m}$. (D) Distance of fungal penetration from surface in C90, Y90 and Y50. Error bar: S.D., $\mathrm{n}=20 .{ }^{* * *} \mathrm{P}<0.001$. 
A Cryo-microtome
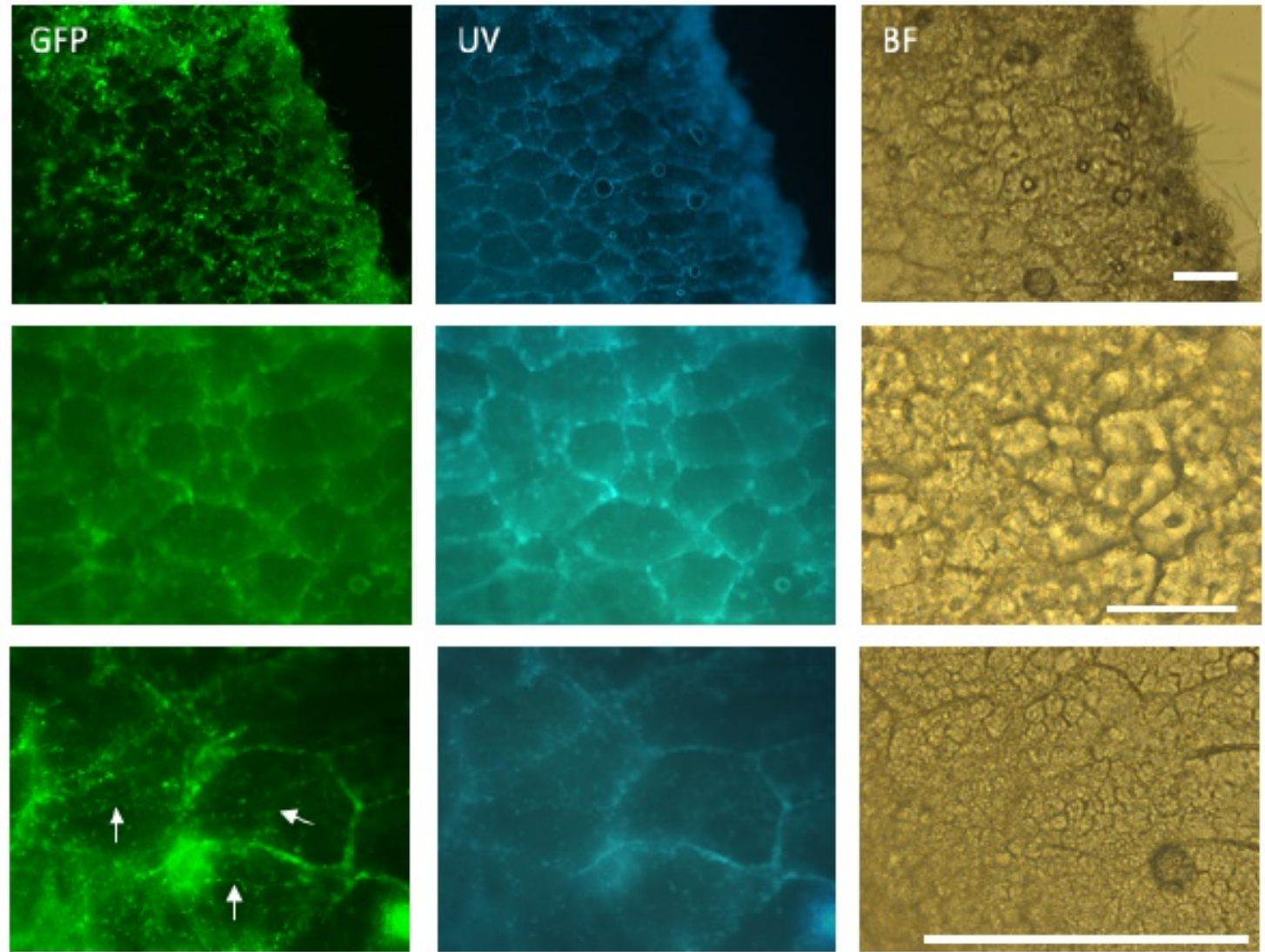

B Confocal-3D
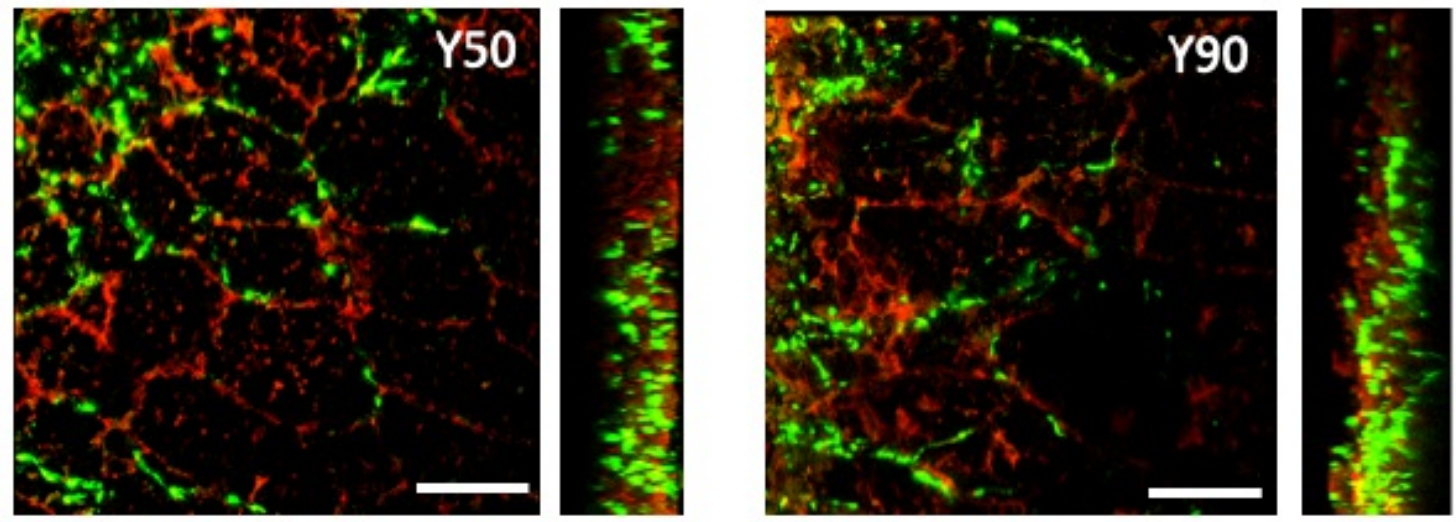

C transparent
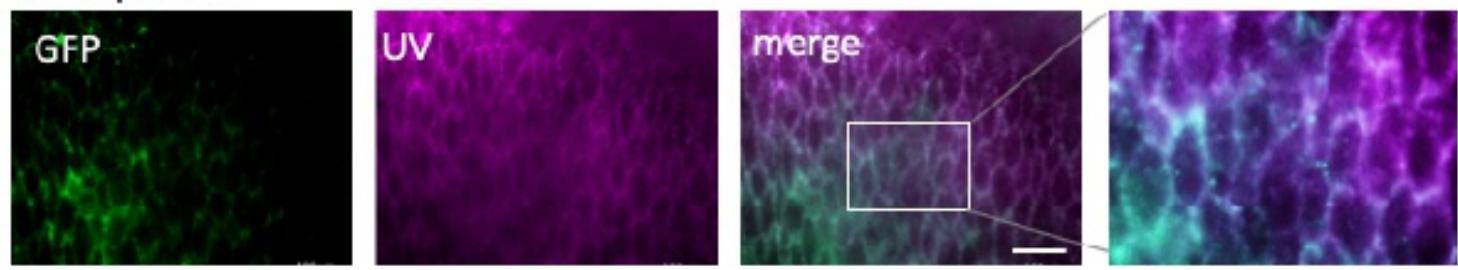

Figure 2

Fluorescent imaging analysis of A. oryzae penetration inter- and intra-rice cells. (A) Fluorescent images of A. oryzae (H2B-GFP) penetration inter- and intra-steamed rice Y50. 
The rice cells were shown by UV and BF (bright field). The sections were sliced by cryomicrotome. Hyphal growth inside of rice cells was indicated by arrows. Scale bars: $50 \mu \mathrm{m}$. (B) Confocal-3D imaging of the koji section from Y50 and Y90. A. oryzae (green), rice cells (red). Movie 1, 2. Scale bars: $50 \mu \mathrm{m}$. (C) The koji were treated with transparent reagent and imaged by fluorescent microscopy. A. oryzae (green), rice cells (purple). Scale bar: $100 \mu \mathrm{m}$.
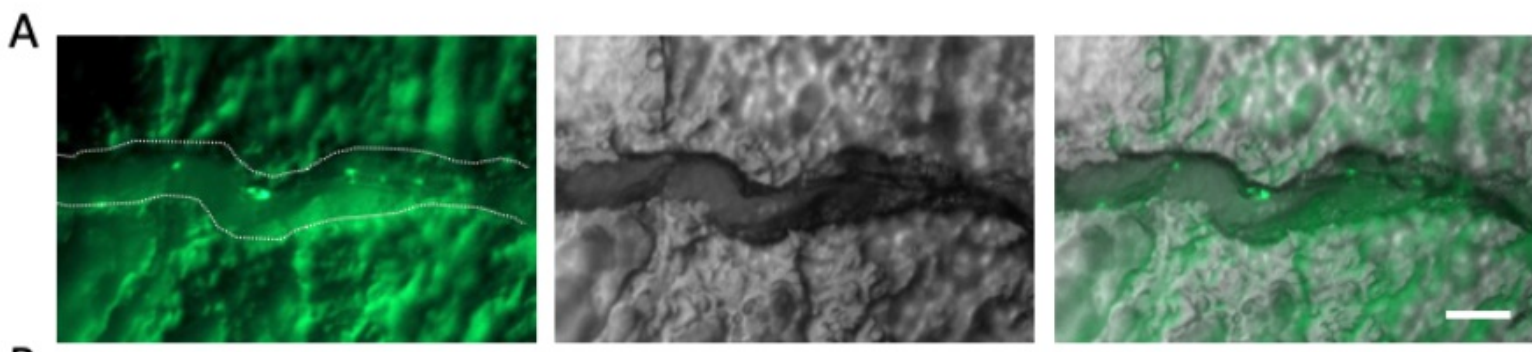

B
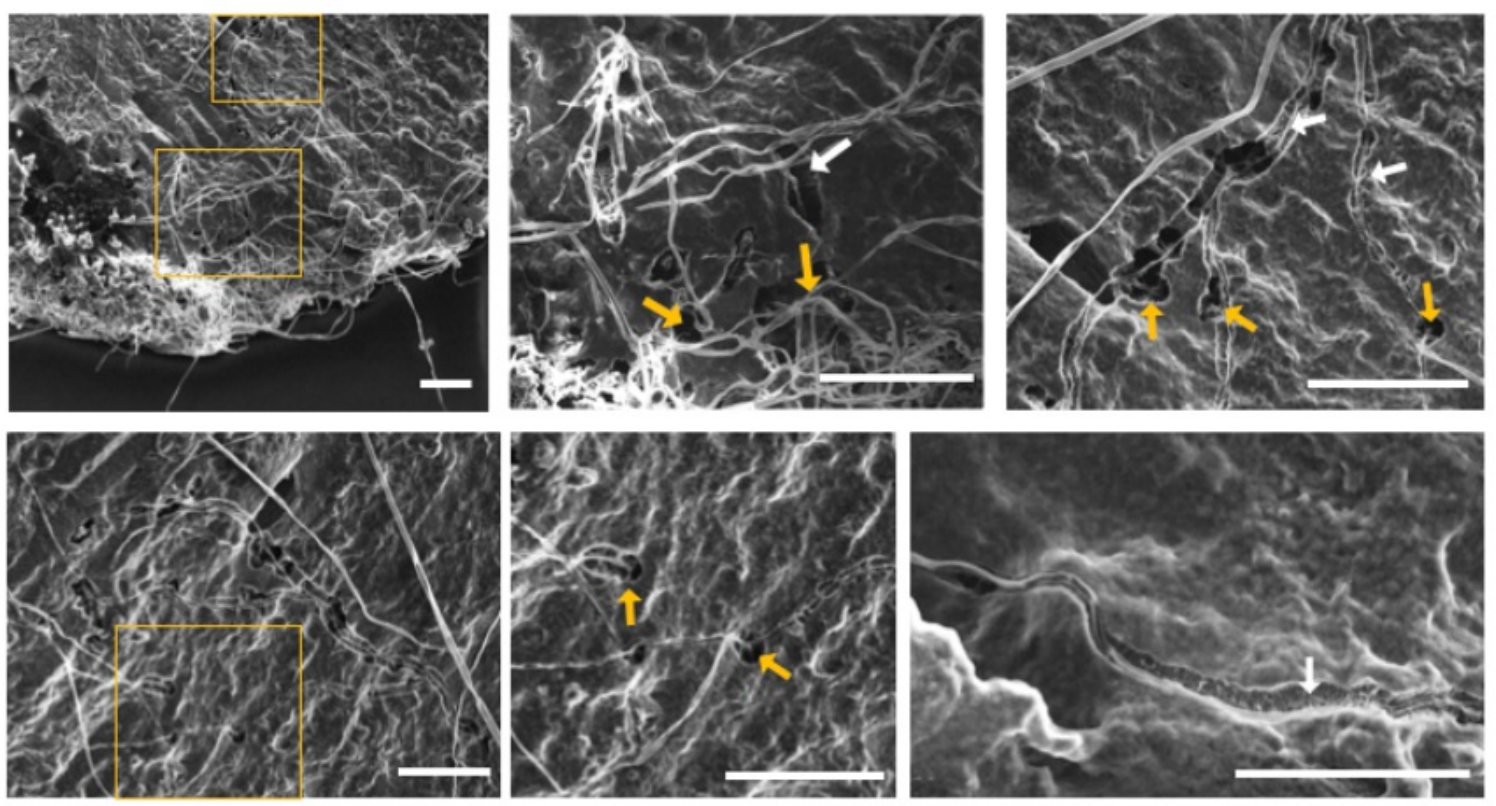

Figure 3

luorescent and SEM imaging analyses of A. oryzae penetration intra-rice cells. (A)

Fluorescent images of A. oryzae (H2B-GFP) penetration intra-steamed rice Y50. Hyphal growth through surrounding space like a furrow in the rice (arrow and dotted line). Scale bars: $20 \mu \mathrm{m}$. (B) SEM images of the koji section. Scale bars: $100 \mu \mathrm{m}$. Cross sections of hyphae from or to holes (yellow arrows). Vertical sections hyphae in ditch (white arrows). 

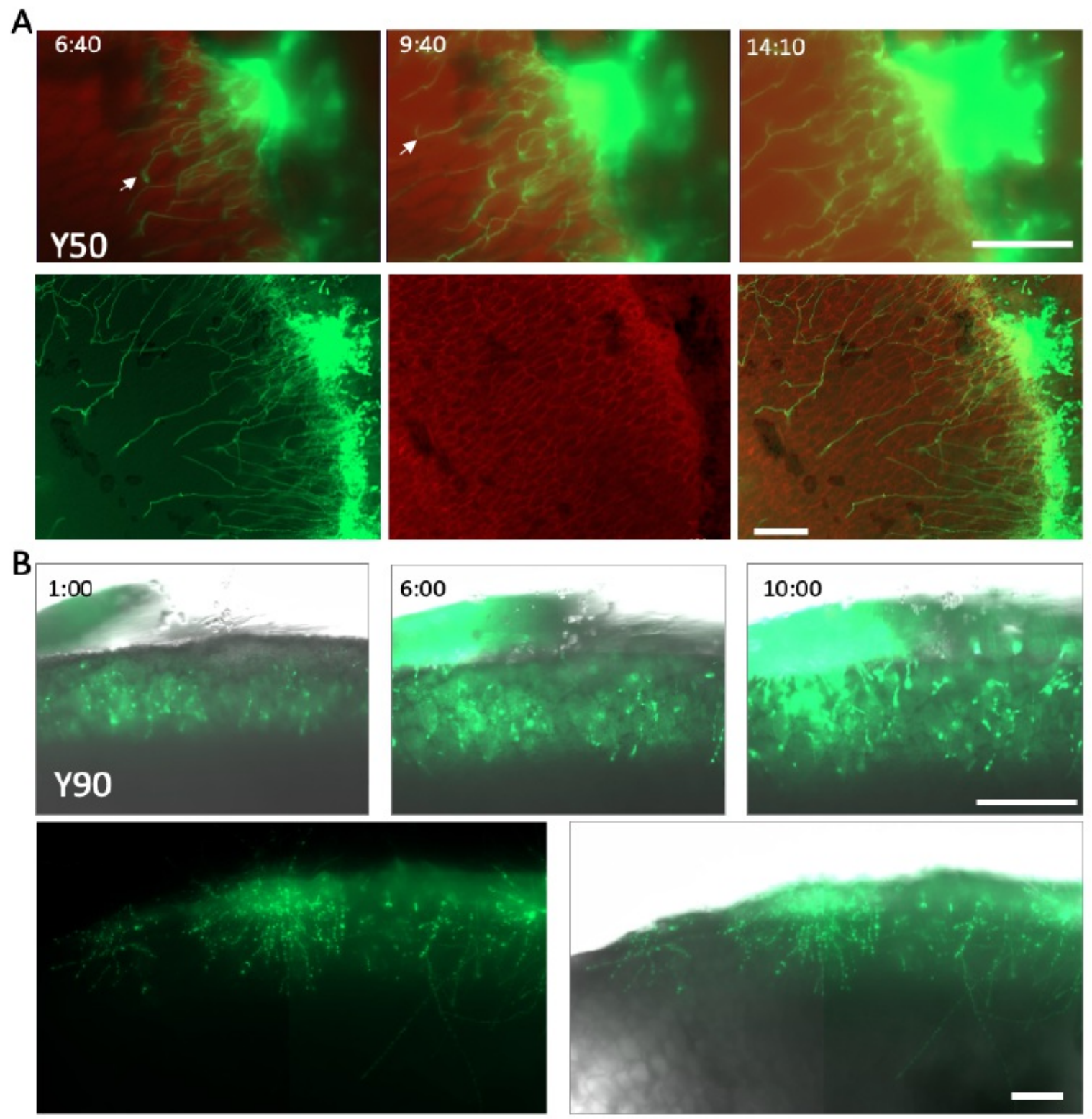

Figure 4

Time-lapse imaging analysis of A. oryzae penetration into steamed rice. The sections of koji

Y50 (A) and Y90 (B), were imaged every 10 or 20 minutes for 14 hours by fluorescent microscopy. Movie 3, 4. The images at three time points were shown (upper), the images at 14 hours were shown (lower). (A) A. oryzae (green), rice cells (red). Some hyphae changed the growth direction after hitting the rice cell shape (arrows). (B) A. oryzae (green), rice cells (bright field). Scale bars: $200 \mu \mathrm{m}$. 
A
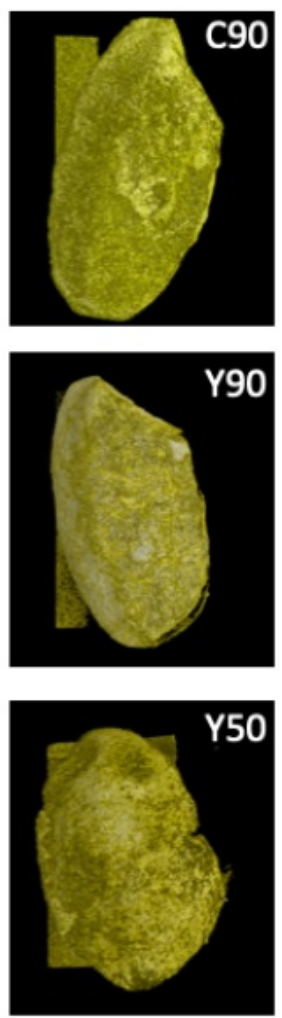
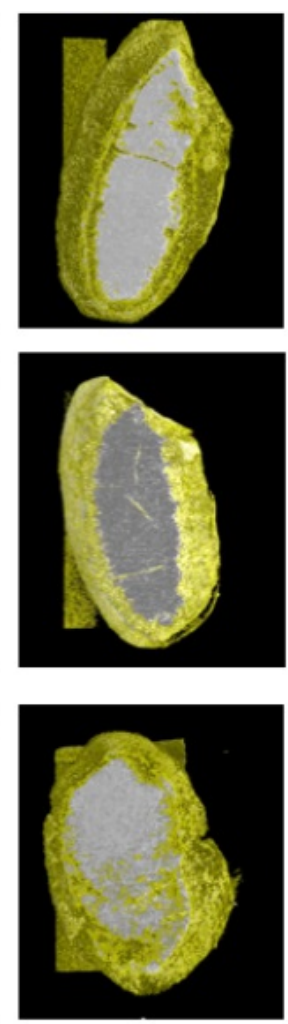
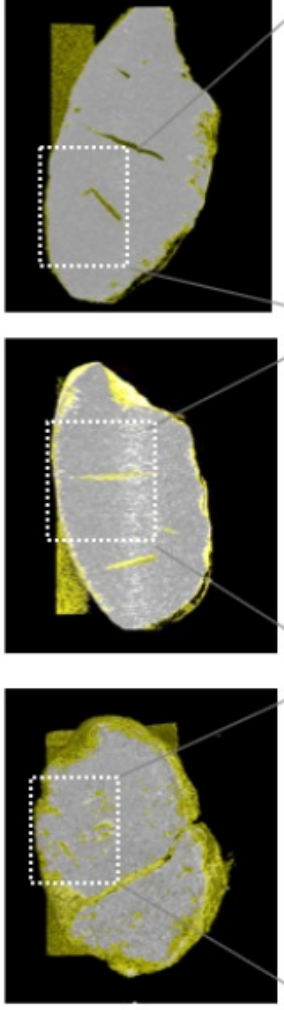

B
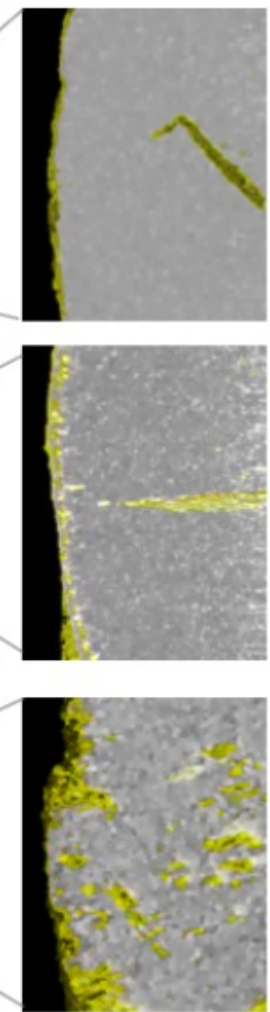

ungus / Rice + Fungus

(vol/vol)

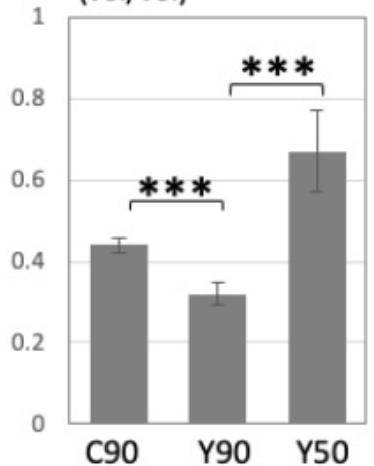

$-\mathrm{Y90}$

- $\mathrm{Y} 90+$ A. oryzae

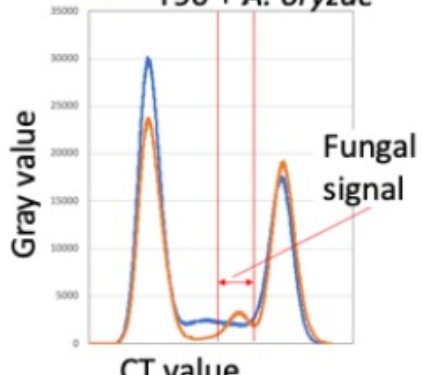

CT value

(X-ray absorption)

Figure 5

X-ray CT analysis of A. oryzae penetration into steamed rice. (A) Image sequence of X-ray

CT analysis in C90, Y90 and Y50, Movie 5-7. The rice and fungus are shown in white and yellow, respectively. (B) Fungal volume was calculated from the 3D data. Error bar: S.D., $\mathrm{n}$ $=3 . * \mathrm{P}<0.05$. The fungal signals were determined by CT value (X-ray absorption).

A class I
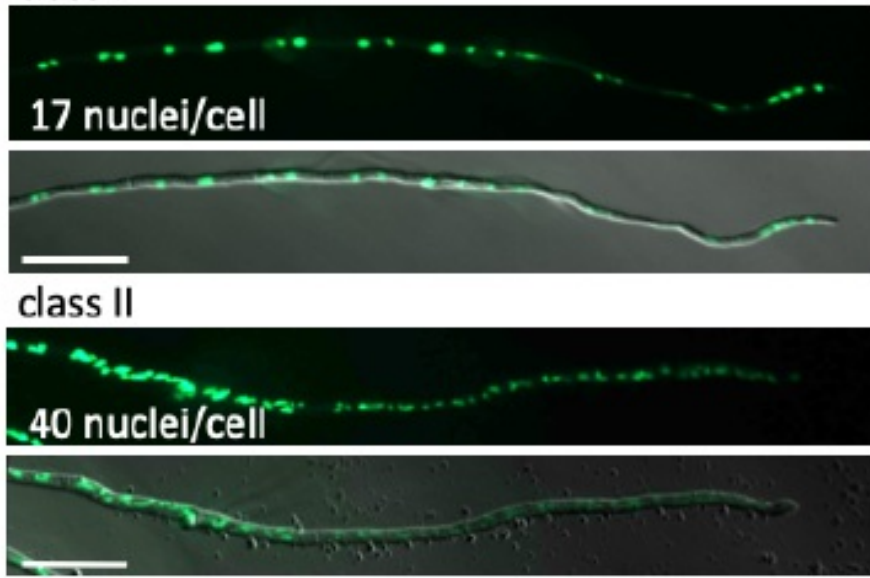

class III

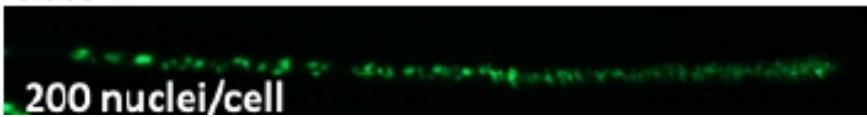

B

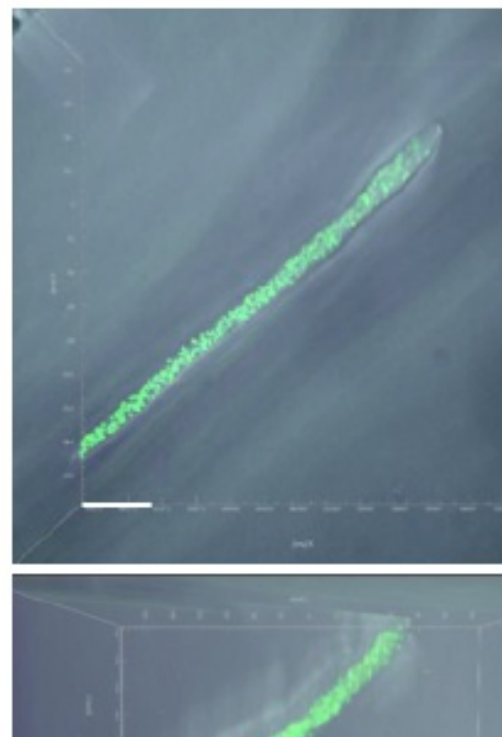




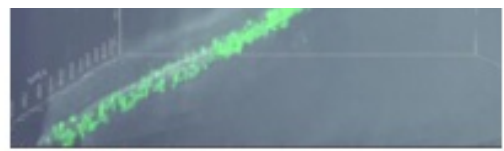

C

A. oryzae

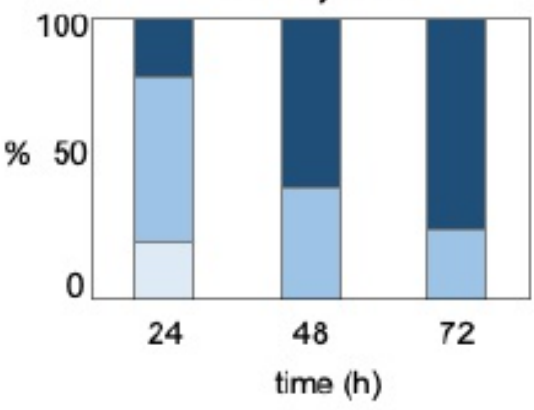

D

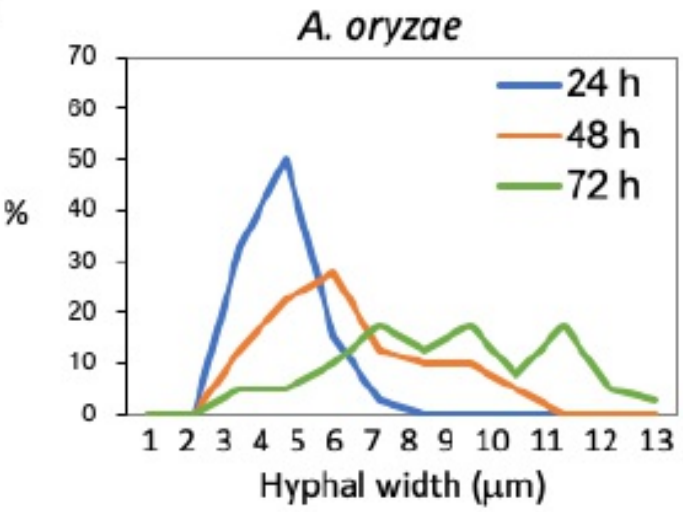

A. nidulans
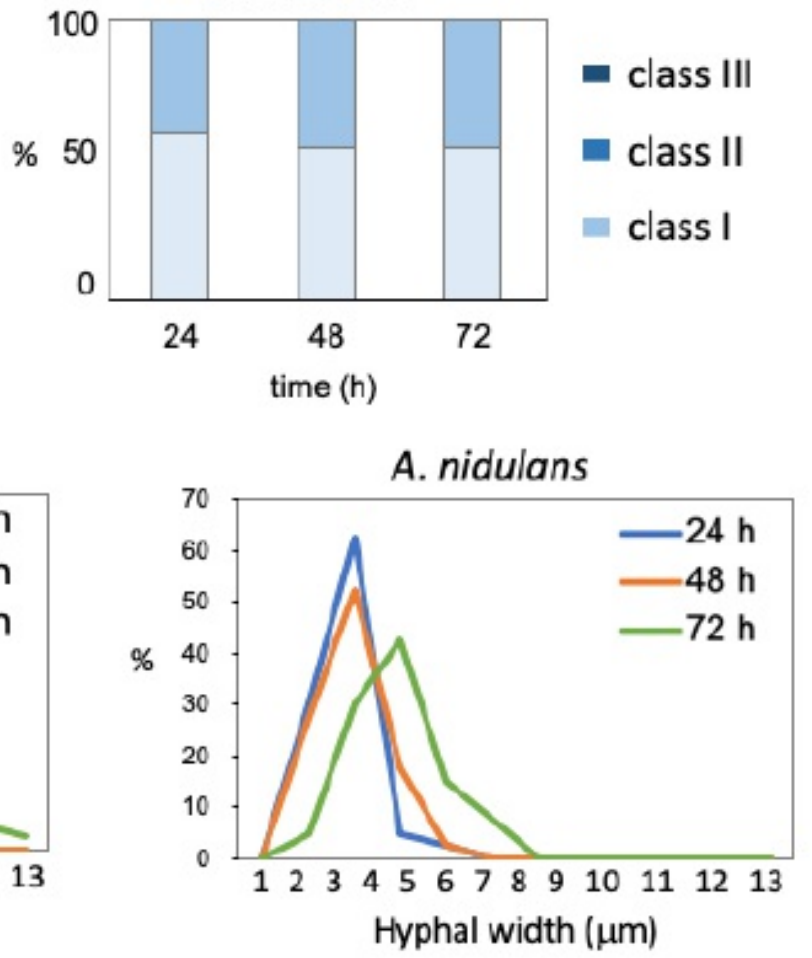

Figure 6

Increase of nuclear number in A. oryzae but not in A. nidulans. (A) Images of nuclei in the tip compartment of A. oryzae. Scale bars: $50 \mu \mathrm{m}$. (B) Confocal-3D imaging of nuclei in the A. oryzae tip compartment. Movie 8 . Scale bar: $40 \mu \mathrm{m}$. (C) Ratio of nuclear distribution pattern classified as class I, II, III (A) in A. oryzae (left) and A. nidulans (right) at 24-, 48- and 72hours growth ( $n=20$ ). (D) Ratio of hyphal width in A. oryzae (left) and A. nidulans (right) at 24-, 48- and 72-hours growth $(n=100)$.

\section{A A. nidulans}
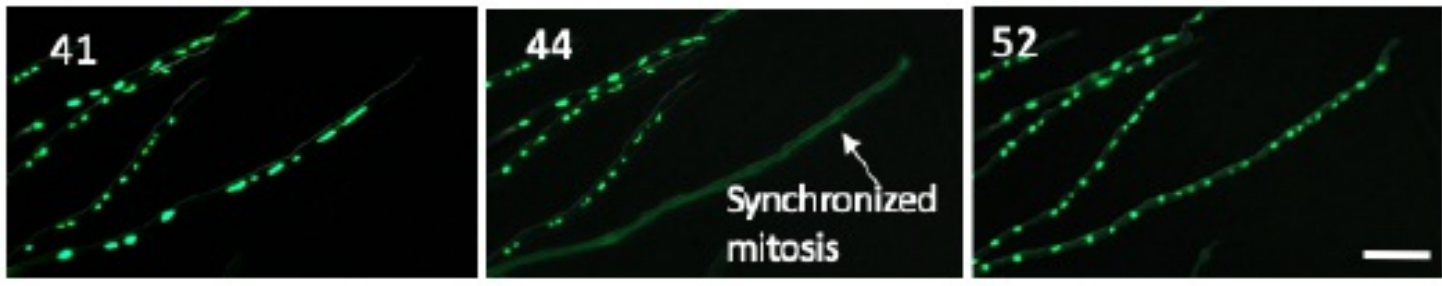

B A. oryzae

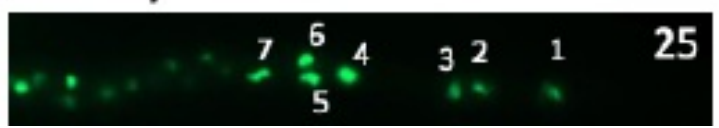




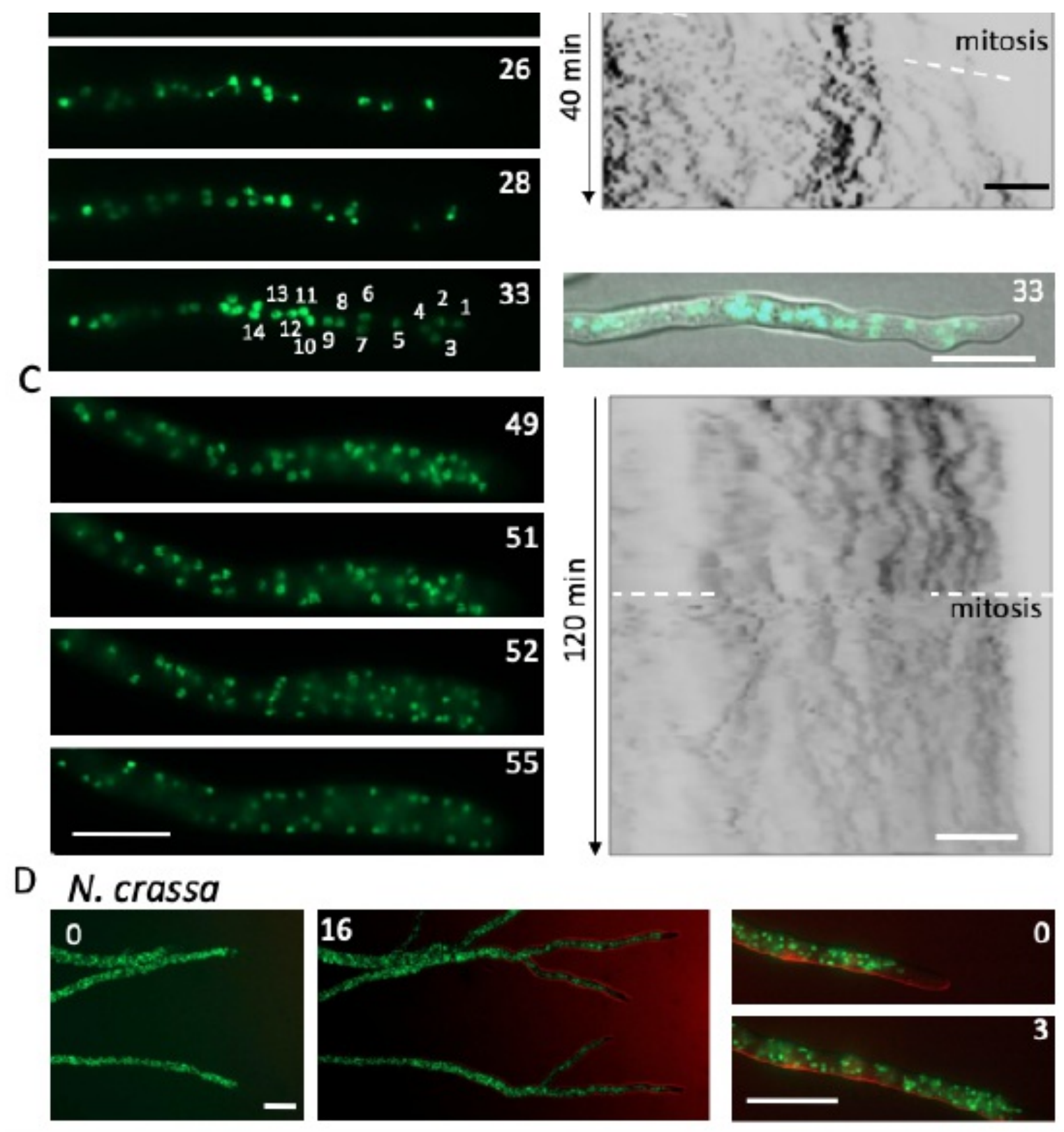

Figure 7

Nuclear distribution and mitosis in A. nidulans, A. oryzae and N. crassa. (A) Image sequence of synchronized mitosis in A. nidulans observed every minute for 3 hours by fluorescent microscopy. Movie 9. (B, C) Image sequence of nuclei in the A. oryzae tip compartment from 1 day incubation (Movie 10) and 3 days incubation (Movie 11). Kymographs of nuclear distribution along the hypha. (D) Image sequence of nuclei in N. crassa from Movie 12, 13. The elapsed time is given in minutes (A-D). (A-C) Scale bars: $20 \mu \mathrm{m}$, (D) Scale bars: $50 \mu \mathrm{m}$. 
This is a list of supplementary files associated with this preprint. Click to download.

Movie 3, Y50 t.avi

Movie 11, Ao mitosis2.avi

Movie 12, Nc t1.avi

Movie 13, Nc t2.avi

Movie 10, Ao mitosis.avi

Movie 5, C90 CT.mov

Movie 7, Y50 CT.mov

Movie 6, Y90 CT.mov

Movie 8, Ao nuc 3D.avi

Movie 9, An t.avi

Supplement table.xIsx

Movie 4, Y90 t.avi

Movie 2, Y90 3D.mov

Suppelemental fig.pdf

Movie 1, Y50 3D.mov 